\title{
REFLEXIONES SOBRE LA GESTIÓN DE PESCA DEPORTIVA ACTUAL EN ESPAÑA
}

\author{
Fernando Torrent Bravo
}

Grupo de Investigación en Hidrobiología. E.T.S.I. de Montes (U.P.M.). Ciudad Universitaria s/n. 28040MADRID. Correo electrónico: fernando.torrent@upm.es

\section{Resumen}

Se realiza un breve resumen de las actividades del Servicio de Pesca en el siglo XX. Se analiza la situación actual, con las transferencias y las nuevas leyes Autonómicas que tratan de regular una supuesta protección, con medios técnicos y humanos mínimos. Se minusvalora en la actualidad, el potencial económico de la actividad deportiva en el Mundo rural. Se propone como alternativa de aprendizaje, la realización de proyectos piloto de demostración que permitan ir adaptando las leyes a una regulación sostenible y enriquecedora del mundo rural.

Palabras clave: Pesca deportiva, Historia Gestión, Futuro, Economía

\section{INTRODUCCIÓN}

La gestión de la pesca deportiva, especialmente en la reciente historia, parece olvidada en España, y en una sociedad como la nuestra, con una industria de turismo espectacular, tenemos que buscar y potenciar alternativas para el ocio y los servicios, y muy especialmente en el desarrollo del mundo rural y litoral. Precisamente el éxito de los campos de golf en nuestro país, es el clima benigno con el que cuenta Clima que beneficiará a todas las actividades que se realicen al aire libre. La pesca, es un potencial de actividad económica en el mundo rural y litoral, muy importante. Existiendo en estos momentos conocimientos, para gestionarlo de manera que genere riqueza en zonas remotas y aisladas y que a la vez, mejore los aspectos de conservación en cuanto a las masas de agua. Esta actividad puede y debe acercar la percepción de estos conceptos de calidad del medio acuático a la sociedad actual.

Precisamente no existen tantos recursos naturales en nuestro país, a los que se pueda recurrir. Y además en los que sea relativamente sencillo actuar. Lo que no hay que olvidar es que su puesta en valor, requiere perseverancia y continuidad en las actuaciones. La naturaleza no se gestiona en el corto plazo, si no que los resultados se obtendrán como resultado de acciones a largo plazo.

\section{ANTECEDENTES E HISTORIA DE LA PISCICULTURA ESPAÑOLA}

No debemos olvidar que la piscicultura surgió como una necesidad del Estado, de repoblar los ríos y las masas de agua interior, que eran una fuente importante de alimentos ricos en proteínas para las poblaciones ribereñas, y que ya se empezaban a dar muestras de agotamiento de los recursos.

En aquella época, el mar se explotaba como fuente inagotable, pero los medios de transporte y conservación, y por ello los mercados eran muy limitados.

A partir de los años 60, en el desarrollo de España, es cuando comenzó a surgir la distribu- 
ción de la pesca y la industria de producción de truchas, que se basó en las tecnologías utilizadas en las producciones de peces de los centros oficiales en funcionamiento.

Vamos a hacer un repaso histórico, que a veces no es tan conocido. La acuicultura moderna surgió en España en 1866, creándose con apoyo entusiasta de la realeza, en la ya histórica primera piscifactoría española en los Jardines del Palacio de La Granja (denominado Laboratorio Ictiogénico de la Granja de San Ildefonso), en Segovia. En la misma época (1863) el emperador austríaco Francisco José, instauró en sus castillos una piscifactoría, así como el rey de Holanda en los suyos y la Reina Victoria, en los jardines del Palacio de Windsor.

Creo interesante reseñar las frases del famoso naturalista Mariano de la Paz Graells, escritas en 1864, dirigidas al Rey, en el "Manual práctico de Piscicultura" que hoy tienen un verdadero valor histórico como embrión de la importante industria de acuicultura española actual.

"Sería de un bien inmenso el que a nuestra Patria reportaría el cultivo racional de sus aguas, hoy casi estériles o incompletas, y casualmente productivas. Esta grande obra es digna, bajo todos los conceptos, de un Rey benéfico que tan decidida protección dispensa a todas las empresas útiles. Y que puede influir para que el Gobierno de la Reina introduzca esta nueva industria en la Península, que le de impulso, que la fomente y proteja en cualquier punto donde se establezca; $y$, dando además el ejemplo práctico, pueda tener la gloria de ser el primero que en España erija un establecimiento de Piscicultura, que venga a ser la escuela nacional de esta utilísima industria, y el semillero que a todos provea de los gérmenes necesarios para poblar de peces comestibles las aguas dulces del territorio español"

Palabras proféticas, que el tiempo convirtió en realidad

Siguiendo con el relato histórico en España, a continuación y en ese mismo año, la familia Muntadas construyó el primer centro privado de acuicultura en el Monasterio de Piedra, cuna de la piscicultura española en 1867 (PARDO, 1953) posteriormente fue arrendada al Estado en 1886, y todavía hoy en día sigue funcionando o al menos hasta el año 2012. Por supuesto está afec- tada por los problemas comunes en nuestros días, asociados con la mala calidad de agua que afecta a tantos cursos de agua en España.

A partir de entonces, en el siglo XX y a lo largo de muchos años el Servicio Nacional de Pesca Fluvial o equivalente se encargó, dentro de la importante gestión de pesca deportiva, de la construcción y puesta en marcha de numerosos centros de piscicultura oficial, que fueron repoblando muy variados cursos de agua. Primero se trabajó con las especies que se conocía su tecnología de producción, donde había especies autóctonas y exóticas, y luego se fueron añadiendo nuevas especies.

En los años 1950 y 1960, con el conocimiento que existía en dicha época, fue un periodo en que se introdujeron muchas especies piscícolas, para su uso como pesca deportiva, y como alimentación de los ribereños. Fundamentalmente estas acciones se justificaban por la construcción de nuevos embalses, que generaban nuevos ecosistemas sin especies con valor comercial, convenientemente adaptadas a ellos. Todo ello, por supuesto realizado con la mejor intención, y que el tiempo ha evidenciado que tuvieron mayor y menor fortuna. Pero siempre existiendo equipos técnicos especializados que actuaban con el mejor conocimiento de la época.

No cabe duda que en la época desarrollista, con la creación de los grandes embalses hidroeléctricos, riegos, ..., y por supuesto con la contaminación de las aguas asociada a dicho desarrollo, cambiaron radicalmente los ecosistemas de los ríos y masas de agua, situación que en el caso particular de la contaminación, está claramente agravada en nuestros días. Las diferencias de la calidad actual de las aguas actuales frente al pasado reciente, convierten a la acuicultura como una herramienta de gestión necesaria, dentro de los sistemas acuáticos que nos rodean en la actualidad. Especialmente si se quiere consolidar una actividad recreativa como la pesca deportiva dentro de nuestras aguas.

Las piscifactorías oficiales se centraron históricamente en salmónidos, principalmente trucha común, pero también salmón atlántico, trucha arco iris, salmón del Danubio y Salvelino. $\mathrm{Y}$ el otro frente de actividad, lo dedicaron a la producción de ciprínidos, y a otras especies (Black bass, Lucio, cangrejo de río, etc). 
No se puede hablar de pesca recreativa y acuicultura sin referirnos al tema de las repoblaciones en ríos o masas de agua. Es un tema candente y debatido, y que al profundizar en el conocimiento de los ecosistemas, en otros países parece superado con una posición más realista e intermedia a los enfrentamientos del pasado. A pesar de ello en España sigue abierto un debate donde ciertas asociaciones conservacionistas se oponen a cualquier tipo de repoblación. La actividad de la repoblación en si no es buena ni mala, solo hay que realizarla de la forma requerida, en el momento adecuado y en el tramo adecuado. No cabe duda de que es una biomasa generada en ambientes cerrados, y sin impacto al medio, que puede contribuir en los tramos adecuados a aumentar enormemente la actividad de la pesca

En España existe una sólida experiencia en las repoblaciones, existiendo criaderos pertenecientes a los Servicios de Pesca desde 1863. Más recientemente los gestionaba el ICONA, hasta que fueron transferidos a las comunidades autónomas en 1990. Pero realmente mucha de la historia y de lo aprendido, no se ha sabido mantener en los Centros de producción.

\section{GESTIÓN DE LA PISCICULTURA, COMO APOYO DE LA PESCA DEPORTIVA}

La gestión actual se debate entre la pura conservación de especies, y la puesta en valor de los recursos naturales. Es posible, con el conocimiento actual, compatibilizar la conservación, con la importancia de poner en valor los recursos naturales, con actividades como la pesca deportiva, que no es que afecten, si no que serán herramientas definitivas en la conservación y mantenimiento de dicha riqueza, en beneficio de las poblaciones locales actuales y futuras.

La inmensa mayor parte del esfuerzo en el pasado, se realizó en la crianza de salmónidos (debido a la riqueza asociada a su pesca, y posteriormente a su consumo), y por ello existe más información generada en España y en otros países del mundo donde los salmónidos son las especies reinas, por lo que el análisis lo vamos a centrar en dicha familia.

En distintos países, y particularmente en España, durante los años 90, se ha puesto en cuestión la filosofía de repoblar sin más como herramienta de gestión, ya que era un método simplista de enfocar el problema. Hoy en día, se le ha dado la importancia que merece a considerar el ecosistema como un conjunto de interacciones y por ello, cuidar el hábitat donde se desarrollan las poblaciones piscícolas, con la misma importancia que cuidar a los propios peces, muy especialmente en lo referido a las cabeceras y en aquellos tramos que permanecen con las características originales.

Hoy se conoce mucho más sobre los peces, y las recientes técnicas moleculares nos han permitido reconocer estirpes propias de muchas cuencas, que antes mediante la apariencia externa no era posible. En la actualidad, existe conocimiento más fundado para manejar las repoblaciones como herramienta de gestión, para ayudar a la puesta en valor de la pesca deportiva, entre otros objetivos.

España es especialmente rica en estirpes de trucha común, por su posición de refugio de la especie en las últimas glaciaciones, lo que se materializa en su supervivencia actual. Posteriormente, al final de la última glaciación, éstas fueron el origen de las truchas que luego fueron repoblando los ríos europeos. Esta característica es una riqueza muy interesante, $y$ tenemos que saber usarla en beneficio de la biodiversidad de los ecosistemas y en beneficio del ejercicio de la pesca, ya que disponemos de estirpes muy originales de una especie mundialmente conocida. Todo ello, siendo conscientes del enorme coste económico de intentar diferenciar poblaciones dentro de instalaciones de producción.

En Estados Unidos, que es un país donde la pesca deportiva tiene una importancia social y económica muy importante, suponiendo en valor, 9 veces los beneficios que aporta la pesca comercial (http://asafishing.org/uploads/2011_ASASportfishing_in_America_Report_January_2013.pdf / )

En dicho país, las repoblaciones, es decir la piscicultura, se consideran de importancia vital para la gestión de las poblaciones piscícolas y el de la riqueza asociada a la pesca deportiva como actividad económica. La gestión está muy condicionada por la tradición de pesca de la región, o la presión social. Como norma general, cada vez más las repoblaciones han ido dirigidas, de una manera biológicamente justificable, a optimizar 
la pesca recreativa. En cualquier caso, no existen dudas que cada uno de los tramos hay que darle el tratamiento que requiere, no pudiendo compararse las ríos que están sometidos a gran presión pesquera cerca de las grandes ciudades, con aquellos tramos que mantienen poblaciones especialmente vulnerables en áreas protegidas.

En Estados Unidos existen 70 criaderos pertenecientes al Estado, y en el año 83, sólo lo repoblado por el Estado, en el caso de especies de aguas frías, era de 256 millones de peces anuales, de los cuales el $30 \%$ eran de talla capturable, el $19 \%$ como jaramugo (ó $1+$ ) y el $51 \%$ como alevín. La iniciativa privada produce enormes cantidades para la repoblación y la pesca, juntando variadas especies con las que se repuebla, se calcula que son unos 600 millones de peces de peces. Cifras muy elocuentes de la industria que manejan

En España, la cría de trucha industrial llegó a producir 120 millones de peces, que suponían 30.000 toneladas cada año, aunque por variadas causas, ha visto mermada su producción significativamente en estos últimos años.

En los datos de 2009, el 70\% de la producción de trucha de ración en USA, de 27.000 toneladas, va dirigida a la pesca recreativa. En España este porcentaje no supera el 5\%. Quedando un largo camino por recorrer, especialmente en un país eminentemente turístico por su clima benigno comparativamente con el del resto de los europeos. En Europa tenemos 24 millones de pescadores, y no pueden venir a pescar a España.

La filosofía de enfrentamiento de los años 80 , donde unos abogaban porque los ríos deben de mantenerse intocados e intentar devolver el estado original previo a la intervención humana, y aquellos que abogaban que debe de tratarse como un recurso más, se ha transformado hoy en día, siendo mucho mas convergente las posiciones, a ello ha contribuido un mejor conocimiento y el poder realizar una gestión más justificada, realizando con planteamientos biológicamente adecuados de las poblaciones.

En España en 1987 se contabilizaban 41 Centros de piscicultura oficiales, gestionados centralizadamente. En esa época se realizaron el traspaso de competencias a las autonomías y desde entonces la gestión de dichos centros ha pasado muchas vicisitudes, que ha originado que mientras maduraban los distintos gestores y formas de gestión, la actividad ha quedado en algunos casos, en punto muerto. Desde el momento en que quedó transferido, ha quedado en manos de las distintas Autonomías, y con gestiones muy diferenciadas. Muy especialmente porque no existe ninguna vía de comunicación oficial y periódica (aunque se van creando poco a poco), con gestión particular de cada autonomía.

La configuración actual de las piscifactorías oficiales dentro de las Direcciones Generales de medio natural, y de otro tipo, ocasiona que muy diferentes técnicos estén ocupados de la gestión piscícola, impidiendo realmente la especialización de los técnicos gestores piscícolas. La realidad es que la normativa, que afecta muy directamente a la actividad de la acuicultura, está redactada por los distintos gestores de las autonomías, y en variados casos, se dictamina una normativa, sin contar con las experiencias debidamente contrastadas de Comunidades vecinas. Estas circunstancias, parecen evidenciar, la necesidad de creación de un canal de comunicación, donde se puedan compartir experiencias, en el beneficio común de todos.

La realidad de la pesca española, es que parece percibirse que va disminuyendo, estando vacíos de peces muchos kilómetros de ríos (con acciones importantes, como la reciente prohibición de pesca en Navarra en 2008, ya vuelta a autorizar, o la reciente pesca generalizada sin muerte en Andalucía en 2007, en Castilla La Mancha y en Castilla León), y de manera continuada reducción del cupo de captura y aumento del tamaño mínimo, que indican claramente que cada día hay menos peces en los ríos.

Las razones son variadas y complejas, pero lo que no cabe duda, es que así como en el caso de la acuicultura marina hay decenas de centros de investigación tratando en investigar nuevos retos y aumentar el conocimiento. En el caso de la acuicultura continental, no existe esa red de centros y mucho menos una correcta coordinación entre los existentes, que permita extender las conclusiones de las investigaciones nacionales e internacionales.

Esta circunstancia está ocasionando una normativa compleja, muy defensiva en la mayor parte de las autonomías y que tiene como conclusión, la redacción de unas normativas, que a pesar 
de ser beneficiosas intuitivamente. Están dejando sin peces los ríos y masas de agua españolas.

Por carecer de competencias los diferentes Ministerios a nivel estatal, existe una labor pendiente fundamental en la gestión de los ríos de España, y consiste en promover la coordinación y mejora de la investigación aplicada. Mediante esos conocimientos adquiridos, se deberán aplicar las más modernas tecnologías y apoyar las iniciativas de transmisión de la información práctica.

Lo anteriormente expuesto, son parte del reciente proyecto de innovación sostenible de la acuicultura continental (www.is-ac.es), centrado en buscar alternativas a los centros de piscicultura continental cerrados en la actualidad, y donde la pesca deportiva y su actividades paralelas, se consideran una actividad muy importante a desarrollar en el futuro.

\section{CONCLUSIONES}

- No se tiene valorado el potencial económico de la pesca recreativa en el mundo rural, ni existe la oferta que permita a pescadores europeos interesarse en la pesca en España.

- La pesca deportiva, a medio plazo no puede estar subvencionada, pues distorsiona el mercado e impide a asociaciones de pescadores y empresas poder ofertarla, al coste real de gestión.

- La acuicultura puede y debe ayudar en la gestión de la pesca deportiva. Se pueden producir los peces extras necesarios en calidad y cantidad para el ejercicio de la pesca recreativa, en los tramos adecuados.

- Creo que solo mediante proyectos pilotos de demostración se podrá salir de la situación actual, por la extremadamente compleja normativa autonómica.

\section{BIBLIOGRAFÍA}

Alonso González, C.; Gortázar, J.; García De JALÓN, D. Y GUTIÉRREZ TeIRA, B.; 2004. Método para la estimación de la fecha de emergencia de los alevines de trucha común (Salmo trutta L.) basado en un modelo de crecimiento para el primer año de vida. En: XII Congreso de la Asociación Española de Limnología y IV Congreso Ibérico de Limnología. Oporto.

Alvarez, J.; 1999. Gestión de la trucha en Navarra; El control de la pesca. Trofeo pesca 78: 64-65.

ArrignON, J.; 1984. Ecología y Piscicultura de las Aguas Dulces. Ed. Mundi-Prensa. Madrid.

Bagliniere, J.L. \& Maisse, G.; 1990. La croissance de la truite commune (Salmo trutta L.) sur le bassin du Scorff. Bull. Fr. Pêche Piscic. 318: 89-101.

COOPER, E.L.; 1970. Management of trout streams. In: N.G. Benson (ed.), A Century of Fisheries in North America: 153-162. Amer. Fish. Soc. Washington.

ChaPMAN, D.W.; 1966. Food and space as regulators of salmonid populations in streams. Amer. Nat. 199: 345-357.

DOADRIO, I.; 2001. Atlas y libro rodo de los peces continentales de España. Dirección General de Conservación de la Naturaleza. Museo Nacional de Ciencias Naturales. Ministerio de Medio Ambiente. CSIC. Madrid.

ElviRA, B.; 1997. El declive de los peces fluviales en España. Ecosistemas 27: 66-71.

Elliott, J.M.; 1994. Quantitative ecology and the brown trout. Oxford University Press. New York.

Garcia De Jalon, D.; 1992. Dinámica de las poblaciones piscícolas en los río de montaña ibéricos. Ecología 6: 281-296.

García De Jalón, D. 1987. Perspectivas en la gestión de la pesca de la trucha en España. Montes 27-30.

García De Jalón, D. y Schmidt, G.; 1995 Manual práctico para la gestión sostenible de la pesca fluvial. AEMS. Madrid.

Garcia De JALON, D.; Tolosana, E. \& Alcalde. F.R.; 1987. Estimación de algunos parámetros poblacionales de la trucha común (Salmo trutta fario L.) en los ríos pirenaicos. Boletín Estación Central de Ecología 29: 51-58.

Garcia De Jalon, D.; Mayo, M.; Hervella, F.; Barcelo, E. \& Villeta, C.; 1990. Pesca Fluvial en Galicia. Bases Limnológicas para su Gestión. Xunta de Galicia. Santiago.

García De Jalón, D.; Mayo Rustaranzo, M. Y Hervella Rodriguez, F.; 1993. Principios y técnicas de la gestión de la pesca en aguas continentales. Mundi-Prensa. Madrid 
García De Jalón, D.; Mayo Rustaranzo, M.; Hervella Rodriguez, F. y Mingo, A.; 1994. Estudio de las poblaciones trucheras de los ríos Tajo, Gallo, Hoz Seca y Cabrillas. Servicio provincial de Montes, Caza y Pesca de Guadalajara. Junta de Comunidades de Castilla-La Mancha.

Garcia MARIN, J.L.; 1992. Diferenciación genética de la trucha común (Salmo trutta) en Espa$\tilde{n} a$. Doctoral Thesis. Univ. Auton. Barcelona.

GARD, R. \& SEEgrist, D.W.; 1965. Persistence of the native rainbow trout type following introduction of hatchery trout. Copeia 1965: 182-185.

HALE, J.G.; 1965. An evaluation of trout stream habitat improvement in a North Shore tributary of Lake Superior. Minn. Fish. Invest. 5: 37-50.

Haury, J.; Ombredane, D. \& Bagliniere, J.L.; 1991. L'habitat de la Truite commune () en cours d'eau. In: J.L. Baglinière \& G. Maisse (eds.), La truite: biologie et ecologie: 47-96. INRA. Paris. págs.

KoHLER, C.C. \& WAYNe, A.H.; 1999. Inland Fisheries Management in North America. American Fisheries Society.

Lasenby, T.A. \& KerR, S.J.; 2001. Brown Trout Stocking: An Annotated Bibliography and Literature Review. Fisheries Section, Fish and Wildlife Branch. Ontario Ministry of Natural Resources. Quebec.

LENNON, R.E. \& PARKER, P.S.; 1959. Reclamation of Indian and Abrams Creeks in Great Smoky Mountains National Park. U.S. Fish Wildl. Serv. Special Sci. Rep. no. 306: 1-22.

LOBON-CERVIA, J.; 1982. Population analysis of the Iberian nase (Chondrostoma polylepis Stein, 1865) in the Jarama River. Vie Milieu 32(3): 139-148.

LOBOn-CERVIA, J. \& Elvira, B.; 1981. Edad, crecimiento y reproducción de la boga de río (Chondrostoma polylepis polylepis Stein, 1865) en el embalse de Pinilla (río Lozoya). Bol. Inst. Espa. Oceanog. VI: 200-213.

Lobon-Cervia, J.; De Sostoa, A. \& Montañes, C.; 1986. Fish production and its relation with the community structure in an aquiferfed stream of Old Castile (Spain). Pol. Arch. Hydrobiol. 34: 333-343.
Martinez, J. \& Garcia De Jalon, D.; 1988. Estudio de las poblaciones trucheras del río Tormes. Ecología 2: 303-313.

MuÑoz GoYANES, G.; 1988. Crónica piscícola continental hispánica. ICONA. Madrid.

ORsBorn, J.F. \& ANDERSON, J.W.; 1986. Stream improvements and fish response. A BioEngineering assesment. Wat. Resour. Bull. 22(3): 381-388.

PARDO, L.; 1950. Apuntes para la historia de la pesca continental española. Ministerio de Agricultura, Dirección General de Montes, Caza y Pesca Fluvial, Instituto Forestal de Investigaciones y Experiencias, Madrid.

PARDo, I.; 1953. Proyecto de innovación sostenible de la acuicultura continental. (www.is-ac.es)

Pla, C. \& García, J.L.; 1998. Genética y conservación de la trucha común española: gestión y conservación de las poblaciones. Trofeo Pesca 66: 40-41.

Pla, C. y García, J.L.; 1998, Diversidad genética y repoblaciones. Trofeo pesca 64: 40-41.

PLATTS, W.S.; 1991. Livestock grazing. In: W.R. Meehan (ed.), Influences of Forest and Rangeland Management on Salmonid Fishes and their Habitats: 389-423. Amer. Fish. Soc. Spec. pub. 19. Bethesda.

Pollock, K.H.; 1994. Angler survey methods. American Fisheries Society, Special Publication 25.

RADOMSKI, P.J.; GRANT, G.C.; JACOBSON, P.C. \& CoOK, M.F.; 2001. Visions for recreational fishing regulations. Fisheries 7-16.

Reeves, G.H.; Hall, J.D.; Roelofs, T.D.; HICKMAN, T.L. \& BAKER, C.O.; 1991. Rehabilitation and Modifying Stream Habitats. In: W.R. Meehan (ed.), Influences of Forest and Rangeland Management on Salmonid Fishes and their Habitats: 519-557. Amer. Fish. Soc. Spec. pub. 19. Bethesda.

Sastre Prats, P.; 2002. Estudio de los métodos de Gestión de Pesca de la Trucha en las diferentes CCAA españolas. Proyecto Fin Carrera. ETSI Montes. Universidad Politécnica de Madrid.

Simmonson, T.D.; LyONS, J. \& KANEHL, P.D.; 1994. Guidelines for evaluating fish habitat in Wisconsin streams. General Technical Report NC-164. United States Department 
of Agriculture. North Central Forest Experiment Station.

STEARNS, S.C.; 1976. Life history tactics: a review of the ideas. Q. Rev. Biol. 51: 3-47.

Templeton, R.G.; 1984. Freshwater Fisheries Management. Fishing News Books Ltd. Farnham.

VIBERT, R.; 1975. Repeuplements des eaux à truites. Bull. Piscicult. franc. 42: 25-48.

VON BertalanFFy, L.; 1938. A quantitative theory of organic growth. Hum. Biol. 10(2): 181-213.
Wesche, T.A.; 1985. Stream Channel Modifications and Reclamation Structures to Enhance Fish Habitat. In: J.A. Gore (ed.), The Restoration of Rivers and Streams. Butterworth Publishers. London.

White, R.I. \& BRYNILDSON, O.M.; 1967. Guidelines for Management of Trout Stream Habitat in Wisconsin. Technical Bulletin No. 39. Department of Natural Resources. Madison. http://asafishing.org/uploads/2011_ASASportfi shing_in_America_Report_January_2013.p df / 